\title{
Math anxiety and executive function: Neural influences of task switching on arithmetic processing
}

\author{
Rachel G. Pizzie ${ }^{1,2}$ (D) Nikita Raman ${ }^{2,3} \cdot$ David J.M. Kraemer ${ }^{2}$
}

Published online: 28 February 2020

(C) The Psychonomic Society, Inc. 2020

\begin{abstract}
Math anxiety (MA) is associated with negative thoughts and emotions when encountering mathematics, often resulting in underperformance on math tasks. One hypothesized mechanism by which MA affects performance is through anxiety-related increases in working memory (WM) load, diverting resources away from mathematical computations. We examined whether this effect is specific to WM or whether the impact of MA extends to an overall depletion of executive function (EF) resources. In this fMRI experiment, we manipulated two separate factors known to impact EF demands - task-switching (TS) and increased WM loadin order to evaluate how MA relates to behavioral performance and neural activity related to mathematical calculations. Relative to a difficult non-math task (analogies), we observed MA-related deficits in math performance and reduced neural activity in a network of regions in the brain associated with arithmetic processing. In response to TS demands, higher levels of math anxiety were associated with a pattern of avoidance and disengagement. When switching from the control task, high math anxiety (HMA) was associated with disengagement from math trials, speeding through these trials, and exhibiting reduced neural activity in regions associated with arithmetic processing. The effects of math anxiety and WM were most pronounced at the lowest levels of WM load. Overall, the results of this study indicate that the effects of MA are broader than previously demonstrated and provide further insight into how EF deficits in MA might impact recruitment of neural resources that are important for successful math computations.
\end{abstract}

Keywords Math anxiety $\cdot$ Working memory $\cdot$ Task-switching $\cdot$ Executive function $\cdot \mathrm{fMRI} \cdot$ Neuroimaging $\cdot$ Arithmetic

Math anxiety (MA), or negative emotions, thoughts, and worries associated with mathematics, has been shown to present a significant challenge for students in STEM fields (Beilock \& Maloney, 2015). Math anxiety is associated with deficits in mathematical processing (Ashcraft, 2002) and achievement (Betz, 1978; Hembree, 1990; Ramirez, Gunderson, Levine \& Beilock, 2013). These deficits may not be attributed to lack of inherent mathematical skill but

Electronic supplementary material The online version of this article (https://doi.org/10.3758/s13415-020-00770-z) contains supplementary material, which is available to authorized users.

Rachel G. Pizzie

Rachel.pizzie@georgetown.edu

1 Department of Psychology, Georgetown University, White Gravenor Hall, 37th and O Streets, Washington, DC 20007, USA

2 Department of Education and Department of Psychological and Brain Sciences, Dartmouth College, Hanover, NH, USA

3 University of Pittsburgh School of Medicine, Pittsburgh, PA, USA are caused by anxiety-related changes in working memory (Ashcraft \& Krause, 2007; Hopko, Ashcraft, Gute, Ruggiero, \& Lewis, 1998). Compared with low MA (LMA) individuals, high MA (HMA) individuals experience an array of anxiety-related behaviors, which likely have an impact on math computations. Previous research has suggested that behaviors associated with math anxiety are characteristic of avoidance (Betz, 1978; Faust, Ashcraft, \& Fleck, 1996; Pizzie \& Kraemer, 2017). Math anxiety impacts working memory by allocating resources that would usually support math computation to systems that process anxious emotion. For example, verbal working memory resources are utilized by negative self-talk and rumination (DeCaro, Rotar, Kendra, \& Beilock, 2010; Maloney, Schaeffer, \& Beilock, 2013). Some of the underlying deficits in performance can be attributed to changes in working memory in HMA individuals (Ashcraft \& Kirk, 2001).

Relatedly, research on executive function indicates that working memory load (i.e., updating) is one of several factors that contribute to demands on executive function resources and that relates to academic performance, including 
mathematical achievement (Bull \& Scerif, 2001; Bull, Espy, \& Wiebe, 2008; Carpenter, Just, \& Reichle, 2000; Friedman \& Miyake, 2017; Miyake \& Friedman, 2012; Miyake, Friedman, Rettinger, Shah, \& Hegarty, 2001; St Clair-Thompson \& Gathercole, 2006). According to a prominent model of executive function (Miyake et al., 2000; Miyake \& Friedman, 2012; Friedman \& Miyake, 2017), the diverse set of cognitive mechanisms and behaviors that make up this construct can be characterized by shifting, updating of information in working memory (WM), and an additional latent factor that is related to these two domains. In in addition to not being wholly separable from shifting and updating, this latent factor is thought to represent aspects of inhibition. Therefore, in this neuroimaging study, we focused on two separable types of executive function (EF) demands - shifting, as manipulated by our task switching (TS) paradigm, and updating, manipulated here by varying the task-relevant WM load - in order to assess their associations with math calculation in MA. In this conceptualization of EF, shifting (TS) and updating (WM load) are considered to be related to one another as aspects of executive function but represent separable components $\mathrm{EF}$ that we have parametrically manipulated in this study. In this way, we aim to better understand - on both behavioral and neural systems levels - how increased executive function demands contribute to underlying brain activity and behavior in increased MA compared with low MA individuals.

To examine the effect of each EF component during mathematical calculation, we explored brain activity while we manipulated EF demands as follows: 1) task switching between variable length blocks of math trials and word-based analogy trials: switching back and forth between task sets requires adopting a new set and initially increases demand on EF resources when one switches to a new task (Friedman \& Miyake, 2017; Liefooghe, Barrouillet, Vandierendonck, \& Camos, 2008; Miyake \& Friedman, 2012), and 2) WM load: increasing the number of arithmetic computations in an orderof-operations task, which increases updating demands as the number of operands increases (Ashcraft \& Krause, 2007). Whereas previous work has manipulated WM load in math anxiety by increasing the difficulty of math problems (Ashcraft \& Krause, 2007b; Faust et al., 1996), here we also manipulated EF through factors that do not necessarily have to do specifically with the math task demands by task-switching.

Math anxiety is associated with avoidance on multiple levels of behavior. At the broadest level, MA individuals avoid taking math classes, which undermines math achievement, and as a result they subsequently shy away from mathrelated careers (Betz, 1978; Hembree, 1990). This pattern of avoidance extends to how HMA students process individual math problems. For example, when HMA individuals were tasked with solving arithmetic problems that were increasingly complex, they showed a speed-accuracy tradeoff (Faust et al., 1996). When WM demand increased, HMA individuals disengaged from these problems and sped through the difficult problems at the expense of accuracy. This initial avoidance response may occur during very early attentional processing of math information (Hopko, McNeil, Gleason, \& Rabalais, 2002; Pizzie \& Kraemer, 2017) and has been demonstrated even when participants are only exposed to numeric stimuli and are not tasked with completing any math computations. For example, HMA individuals showed difficulty with executive function when a Stroop task was completed with numerals, indicating that even incidental exposure to mathematical information is related to executive function and inhibitory mechanisms (Hopko et al., 2002). Using an attentional deployment paradigm, increased MA was associated with a disengagement bias for math information (Pizzie \& Kraemer, 2017), such that HMA individuals avoided looking at mathematical expressions even when they knew they would never be asked to solve them.

Deficits associated with math anxiety are exacerbated by increased WM load, pointing to diminished EF and WM resources as a hypothesized mechanism by which anxiety interferes with math performance (Ashcraft, 2002; Beilock, 2008; Beilock \& Ramirez, 2011). For example, adding "carry" operations to arithmetic problems to increase the difficulty resulted in deficits for HMA individuals (Faust et al., 1996). HMA individuals also show an enhanced effect in which as the number of operands in an arithmetic problem grows larger (and also the magnitude of the numbers calculated increases), HMA individuals show increased errors and increased response time (Ashcraft \& Krause, 2007). Overall, MA is related to executive function and working memory resources through a deficient inhibitory mechanism, such that taskirrelevant anxious thoughts disrupt cognitive resources (DeCaro et al., 2010; Hopko et al., 1998). Moreover, increased MA was associated with increased attention to distractors and lessened ability to devote WM resources away from distracting negative thoughts (Hopko et al., 1998). However, this interpretation suggests that other executive functions besides WM also should be impacted by MA, because these functions, such as task switching, also are known to depend on inhibitory mechanisms (Miyake \& Friedman, 2012).

In the current experiment, we manipulated EF in two ways, by modulating shifting through task-switching and updating, a concept related to working memory load, in order to gain a better understanding of how these changes in cognitive resources would be associated recruitment of neural systems important for mathematical computations. Additionally, we examined how manipulations of WM load would be associated with avoidance behaviors in MA. Math anxiety is associated with increased avoidance (Faust et al., 1996; Pizzie \& Kraemer, 2017), and WM deficits are created by increasing cognitive load (Ashcraft, 2002). Previous research has suggested that math anxiety is separately associated with both avoidance and working memory deficits, but our experiment 
considers both behaviors together. This consideration is important, because increased pressure on WM resources might be associated with two different patterns of behavior, resulting in increased engagement due to effort on the task or increased disruption due to avoidance or distracting thoughts (Ramirez, Shaw, \& Maloney, 2018; Suárez-Pellicioni, Núñez-Peña, \& Colomé, 2015). If deficits are attributed to utilizing more working memory resources for inefficient strategies, we would expect increased effortful engagement with the task at hand, attempting to compensate for the additional draw on executive function resources. In terms of neural activity, in this case, we would predict increased neural activity in regions of the brain associated with mathematical calculations for increased math anxiety. However, if deficits are caused by WM being disrupted by distracting ruminations or negative emotion, we would expect these deficits to be associated with avoidance or disengagement. In this case, we hypothesized that increased math anxiety would be associated with decreased activity in regions of the brain associated with mathematical calculations, due to disrupted processing and avoidance of math. Thus, in the present experiment, we examined how increasing demand on executive function resources would be associated with variation in math anxiety, taking into consideration these two hypotheses for how increased demand on EF and WM resources would be related to engagement or avoidance in more math anxious individuals.

In the present experiment, we utilized two workingmemory-intensive tasks to exacerbate the demand on working memory resources: order of operations arithmetic problems (math), and analogies. In this view, the "order of operations" math task utilized in this experiment is very appropriate as a measure of working memory: participants must make multiple calculations in a stepwise manner, holding multiple pieces of information in mind while additional calculations are made. In addition, these participants must hold these calculations in mind during the initial calculation period and then wait for the subsequent appearance of the answer screen, holding their own calculated answer in mind and comparing it to the answer presented on the screen. In addition, with each additional operand, it adds another item and calculation to the working memory load. In this way, we utilize the number of operands in each problem as a method to operationalize difficulty and working memory load, with each additional operand adding to the working memory load of the problem. Similar to the order of operations task, we argue that the analogy task presented to our participants also critically relies on working memory to a similar degree to the arithmetic task. In this task, participants must evaluate the relationships between multiple words, and then hold this information in mind while they wait for the answer screen, evaluating whether the word presented on the subsequent answer screen is in line with the analogical relationship presented on the previous screen. Thus, both the order of operations task and analogical reasoning task presented to our participants both include similar levels of working memory demands, both involve manipulating multiple pieces of information, and temporarily holding that information in mind before it can be utilized to complete the task.

However, only limited research has explored how brain activity is associated with MA and WM (Lyons \& Beilock, 2012a). To date, very few studies have investigated brain activity in adults while HMA individuals are actually performing math computations (Pletzer, Kronbichler, Nuerk, \& Kerschbaum, 2015; Pizzie, McDermott, Salem \& Kraemer, 2019; Suarez-Pellicioni, Nùñez-Peña, Colomé, 2013; NúñezPeña, Tubau, Suarez-Pellicioni, 2017). Most of the previous neuroimaging literature focuses on younger populations (Supekar, Iuculano, Chen, \& Menon, 2015; Young, Wu, \& Menon, 2012), and brain activity observed during anticipatory periods (Lyons \& Beilock, 2012b; Lyons \& Beilock, 2012a), or when participants are merely exposed to mathematical information and not asked to compute answers (Pizzie \& Kraemer, 2017).

In this paradigm, we manipulated EF task demands utilizing task-switching, examining how increasing pressure on $\mathrm{EF}$ resources would disrupt cognitive functioning in increased MA. In a set of exploratory analyses, we also explored how increasing working memory load, as referenced by task difficulty, would additionally be associated with cognitive load EF across the spectrum of MA. We would expect that HMA individuals would show decrements in task performance that are consistent with avoidance behavior compared with low math anxious (LMA) individuals (these individuals score so low on a math anxiety measure that we may consider them to be "non-math anxious"). Task switching and increasing the difficulty of math problems will add additional pressure on the executive function resources of HMA individuals, through additional pressure on shifting and updating resources, respectively (Sharp, Miller, \& Heller, 2015). Therefore, we would expect that both of these factors will result in performance deficits and avoidance. We would expect that HMA individuals would experience additional demands on executive function when switching into a math task (compared with switching into an analogy task) due to the increased anxiety experienced for math. This increased anxiety for math adds pressure onto the executive function demands created by adopting a new task set, as well as the demands on working memory created by increased anxiety. On a neural level, we hypothesize that increasing WM load would be associated with variability in activity in the brains of HMA individuals due to avoidance behaviors. This avoidance would lead to decreased activity compared with LMA individuals (Young et al., 2012). We specifically selected a network of regions hypothesized to be selective for neural activity related to arithmetic computations. In this way, we examined how executive function demands alter functionality in brain regions subserving arithmetic and how MA is associated with this 
neural processing. This study represents an important investigation into the brains and behaviors of HMA individuals, giving us insight into how intensifying demands on executive function will be associated with deficits in math computation in increased math anxiety. We predict that variability in MA will be associated with increased executive function demand, disruption of these resources by increased anxiety, and reduced math computation in the brains of HMA individuals. Furthermore, the pattern of behavioral results will add insight into whether HMA individuals experience increased avoidance when faced with mathematics in an increasingly difficult scenario.

\section{Method}

Participants Fifty-three undergraduate participants were recruited on the basis of their extreme scores on the MARS (Suinn \& Winston, 2003). Participants were drawn from a subject pool composed of undergraduate students enrolled in introductory psychology and neuroscience classes. Fifty-three participants were recruited on the basis of their high and low scores on the MARS $\left(N=488,62 \%\right.$ female, $M_{\text {age }}=19.45$ years, $M_{\text {MARS }}=2.26, S D_{\text {MARS }}=0.56$, MARS range: $1-5$, Extreme scores were $\pm 0.7 \mathrm{SD}$ away from mean ${ }^{1}, M A R S$ LMA range $=1.00-1.84$, MARS - HMA range $=2.66-4.67$ ) Math anxiety scores significantly differed between the high and low groups, $t(35.40)=18.25, p<0.0001$. A total of five participants were excluded from the analysis: one for a technical error during the first fMRI run, one for excessive motion in the scanner ( $>4 \mathrm{~mm}$ absolute motion), one for claustrophobia, one for signal dropout, and one for accidental scan invalidation due to an alarm. The final sample included 48 subjects $\left(\mathrm{M}_{\text {MARS }}=2.35, \mathrm{SD}_{\text {MARS }}=0.85\right): 24$ low math anxious participants (LMA; LMA range: $1.0-1.83, M_{\text {MARS }}=1.53, S D_{\text {MARS }}=$ $0.18, M_{\text {age }}=20.13,58 \%$ female) and 24 high math anxious participants (HMA; HMA range: $2.70-4.46, M_{\text {MARS }}=3.11$, $S D_{\text {MARS }}=0.38, M_{\text {age }}=19.13,71 \%$ female). Despite the slightly unequal distribution across MA groups, there were no average differences in self-reported MA between females $\left(M_{\text {MARS }}=2.42\right)$ and males $\left(M_{\text {MARS }}=2.24\right), t(38.59)=0.73, p$ $=0.47$. All were screened for MRI safety precautions, and all experimental measures were approved by the Dartmouth Committee for the Protection of Human Subjects (CPHS). All participants provided written, informed consent, and

\footnotetext{
${ }^{1}$ These cutoffs were chosen to include approximately $10 \%$ of the population of participants in the subject pool at either end of the range of MARS scores (approximately $40 \mathrm{HMA}$ and 40 LMA participants). This range also was chosen because these scores would create groups of participants with MARS scores that were significantly different from one another, and significantly different from the mean of scores (comparing to a mean of 2.26, LMA: $t(487)=16.56, p<0.001$; HA: $t(487)=15.78, p<0.001)$.
}

participants received either course credit or monetary compensation for their involvement in the study.

Power Analysis. To estimate the sample size for this study, we used a previous math anxiety experiment to estimate the number of participants who would be needed for sufficient statistical power for the behavioral analyses in this study, calculated using G*Power 3.1 (Faul, Erdfelder, Lang, \& Buchner, 2007). A previous study examined behavioral disengagement across levels of math anxiety (Pizzie \& Kraemer, 2017). We used the behavioral effect size of $\eta_{p}{ }^{2}=0.08$ (p. 103 ) as an estimate to examine the interaction between math anxiety and behavioral disengagement in the present study. Given this effect size $\left(\eta_{p}{ }^{2}=0.08\right.$, Effect size $f=0.295, \alpha=$ 0.05 , and estimated power $=0.80$, and correlation among repeated measures $=0.2$ ), we would expect to recruit 28 individuals to achieve $82 \%$ power for a repeated measures ANOVA, estimating statistical power for a within- and between-subject interaction (e.g., estimating math anxiety effects across 4 within-subject categories of stimuli). Because our sample included 48 participants for data analysis, we expect that we have adequate statistical power to assess these behavioral effects.

Task. Participants solved math and analogy problems while in the fMRI scanner. Subjects completed 2 sets of 80 trials each of math and analogies for a total of 160 trials over the course of $2 \mathrm{fMRI}$ runs. To evaluate task switching, trials were presented in a pseudo-randomized sequence designed to present trials in sequences of a minimum of two and a maximum of eight of the same type of trial (either math or analogy) per block (Fig. 1A). The overall order of trials in the experiment included sequences of varying length of math and analogy trials. This order was designed so that subjects could not anticipate when a switch between math and analogy trials would occur. The analogy and math sequences were counterbalanced for difficulty (measured by accuracy in pilot data) across trial types. In our exploratory analyses, math trial difficulty, measured by number of operands in the order of operations problems, also was counterbalanced across trial categories.

Each trial in the paradigm was designated as switch, nonswitch, or null (Fig. 1A). "Switch" trials were defined as initial trials of one type (math/analogy) immediately following trials of the other type. Stated another way, switch trials are defined as the first "different" trial in a sequence, or the initial trial in a block. "Nonswitch" trials were designated as either the fourth, fifth, or sixth of the same trial type (math or analogy) in a sequence (but were not the last trial). Nonswitch trials allowed us to examine if trials later in a sequence differed from the initial trials. Null trials were defined as any trials that were not designated as either switch or nonswitch: the second and third trials, or the last trials, in any given sequence of math or analogy trials. Null trials were not analyzed in the behavioral analysis and were accounted for as a regressor of no interest in the fMRI analysis. A total of 15 
a

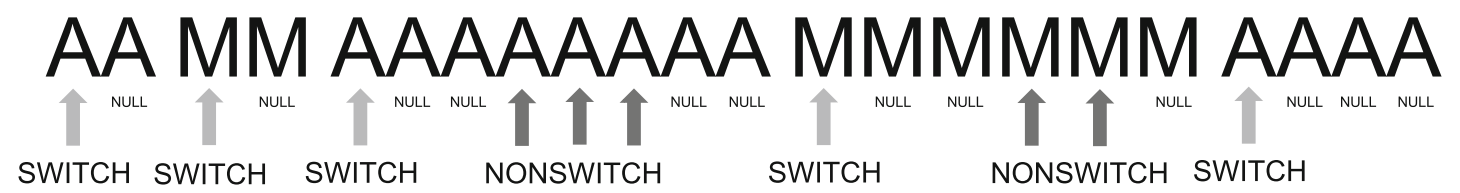

b

Math Trial

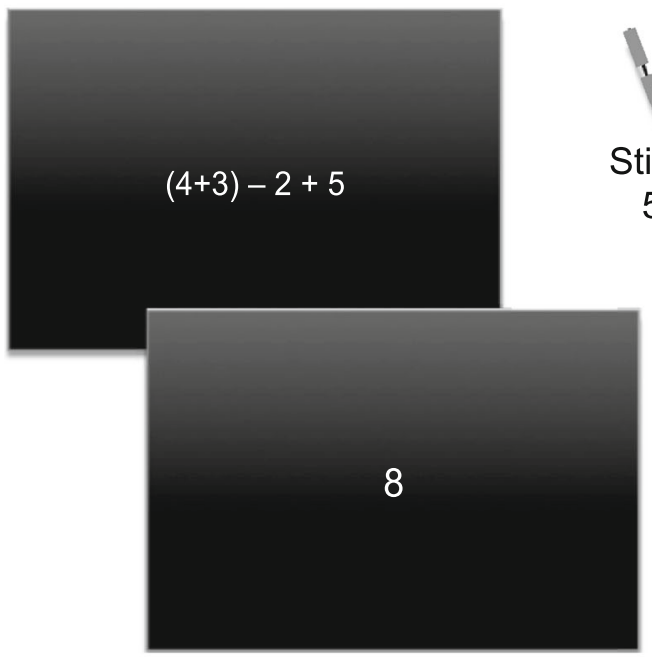

Button press: Correct/Incorrect?

Fig 1. Trial cadence and switching. A) Trial sequence including "Switch," "Nonswitch," and null trials. "Switch" trials include the first trial in a block of math (M) or analogy (A) trials. "Nonswitch" trials were drawn from trials later in the block- the $4^{\text {th }}, 5^{\text {th }}$, and $6^{\text {th }}$ trials in a sequence of trials (but only if they were not the last trial in the sequence). All other trials were "null" and were not analyzed in the behavioral results

math switch trials, 15 analogy switch trials, 24 math nonswitch trials, and 24 analogy nonswitch trials was presented to each participant. All participants received the same pseudo-randomized order so that the number of switch and non-switch trials could be balanced across math and analogy conditions.

On each trial, participants were shown a question (either an incomplete analogy or an order-of-operations math problem) for 5 seconds (2 TRs; Fig. 1B). They were then presented with a possible answer for 5 seconds. On the answer screen, participants were given the task of indicating whether the answer was correct or incorrect. Math trials were arithmetic problems that could be solved by using an order of operations technique (e.g., " $(8 \times 9) \div 3 \times 9$ ") and were drawn from a random problem generator (TheTeachersCorner.net worksheet generator; incorrect answers that were presented were produced by solving the problem "incorrectly" in a linear fashion and ignoring the order of operations information). Participants were asked to solve the problem for 5 seconds and were subsequently asked to indicate whether a number presented on the answer screen was the correct solution to the problem. Response times and accuracy data were
Analogy Trial

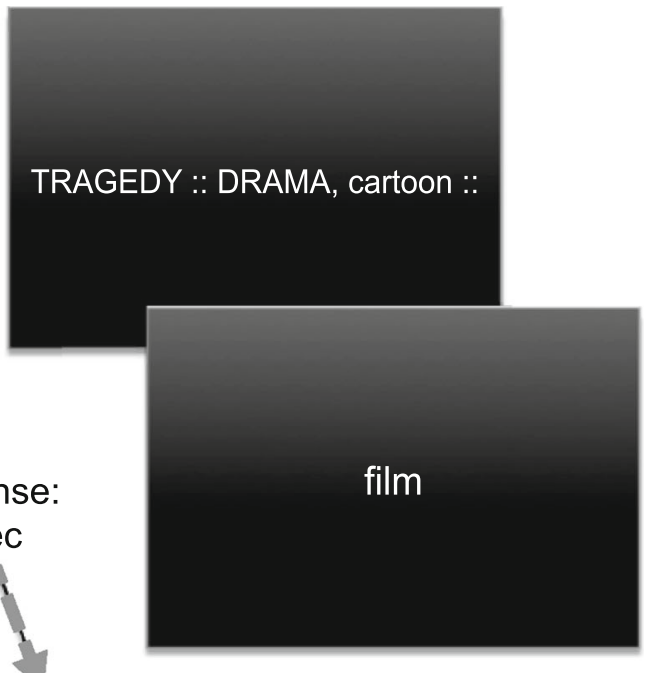

Button press: Correct/Incorrect?

and were entered as a regressor of no interest in the fMRI analyses. Participants completed 15 switch trials and 24 nonswitch trials for each condition. B) Trial cadence indicating math (left) and analogy (right) trials. Trials were shown in randomized blocks, creating sequences of varying length, as depicted in $\mathbf{A})$.

recorded during this answer screen (which occurs after the initial deliberation period of 5 seconds). For analogies, participants viewed an incomplete analogy for 5 seconds (e.g., "DEFERENCE :: RESPECT, affection ::") and then evaluated a word presented on the answer screen for 5 seconds to determine whether it correctly completed the analogy. Analogies were drawn from previous examples of problems used on the Graduate Record Exam.

Questionnaires. After completing the fMRI portion of the experiment, participants completed a series of computerized questionnaires assessing emotion regulation abilities and various types of learning anxieties. Questionnaires were completed on a computer using Qualtrics software (www.qualtrics. com). In all of the questionnaires, subjects responded to statements using a Likert scale. Positive and negative affect was assessed using the Positive and Negative Affect Schedule (Watson, Clark, \& Tellegen, 1988). The Spielberger StateTrait Anxiety Inventory was used to assess trait anxiety (STAI; Spielberger, 2009). Test anxiety was measured using the Test Anxiety Inventory (TAI; Spielberger \& Spielberger, 1980). Writing anxiety was assessed with the Daly-Miller Writing Apprehension Test (Daly \& Miller, 1975). Emotion 
regulation techniques, including emotional reappraisal and suppression, were assessed using the Emotion Regulation Questionnaire (Gross \& John, 2003). Participants also provided basic demographic information including age, race, and gender. Following administration of the surveys, subjects were debriefed on the purpose of the study, thanked for their participation, and compensated for their time.

Image Acquisition: fMRI Parameters. Participants were scanned at the Dartmouth Brain Imaging Center in a $3 \mathrm{~T}$ Philips Intera Achieva MRI scanner with a 32-channel head coil. A structural scan was performed using a T1-weighted image (approximately $1-\mathrm{mm}^{3}$ resolution). The Philips FFE EPI sequence was used to acquire functional images covering the whole brain, using 3-mm slice thickness for a total of 42 interleaved axial slices. This study used an 80- x 80-pixel matrix in a $240 \mathrm{~mm}^{2}$ field of view (Flip angle $=90$ degrees, $\mathrm{TE}=35 \mathrm{~ms}, \mathrm{TR}=2.5 \mathrm{sec}$ ). Due to the rapid nature of the task, fixation was limited to periods at the beginning and end of the task (7 TRs each). In functional runs, 332 TRs of functional activity were collected during each of two runs, such that the task took approximately 27 minutes to complete.

Analyses. Neuroimaging analysis was conducted using standard practices in FSL v. 6.00 (Jenkinson, Beckmann, Behrens, Woolrich, \& Smith, 2012). Neuroimaging preprocessing involved motion correction and registration of functional and structural data using FSL's MCFLIRT and brainextraction of structural and functional data using bet2. Functional data were filtered using a high pass filter (100 $\mathrm{Hz}$ ), pre-whitened, smoothed using a 3-mm gaussian kernel, and a slice timing correction was applied. Functional data were registered to the standard MNI brain $(2 \mathrm{~mm})$ and were convolved using a double-gamma hemodynamic response function. Modeling of mixed and fixed effects models in was done using FSL's FEAT. Data were extracted from ROIs using FSL's FEAT. In whole-brain analyses, clusters of activity are corrected for multiple comparisons using FSL's cluster correction algorithm and have a threshold of $\mathrm{z}>2.3$. $(p<0.05$, corrected).

Behavioral analyses using linear mixed models and linear models were completed using R and RStudio (https://www.rproject.org/). Linear mixed models were completed comparing a categorical fixed factor for stimuli, contrasting math and analogy trials, as well as comparing a categorical fixed factor for task-switching category. In behavioral analyses, RT for all trials was used. Math anxiety was evaluated using residuals from a regression between math anxiety (MARS scores) and test anxiety (TAI), allowing us to examine the effects of MA while accounting for test anxiety (MA-TAI scores), which are used throughout these analyses. Although previous research has controlled for trait anxiety when analyzing math anxiety scores, here we chose to control for test anxiety in our math anxiety scores (MA-TAI scores). Test anxiety is closely related to the MARS, and these scores are largely covaried with one another (Pizzie \& Kraemer, 2019). In a linear regression predicting MARS scores, TAI is strongly associated with MARS scores, $F(1,46)=55.4, p<0.0001$, adjusted $R^{2}=0.53$. When we add trait anxiety to this model, STAI-trait scores (beta estimate $=0.3, p=0.18$ ) are not a statistically significant predictor above and beyond the variance accounted for by TAI (beta estimate $=0.90, p<0.001$ ), $F(2,45)=29.14, p<0.0001$, adjusted $R^{2}=0.55$. To further illustrate this point, we compared the variance accounted for by these two models predicting math anxiety (test anxiety, and test anxiety + trait anxiety) using the Anova function in R. This model comparison allows us to compare whether adding trait anxiety to the model of test anxiety accounts for significantly more variance than just utilizing test anxiety to predict math anxiety. In predicting math anxiety scores, we do not find that adding trait anxiety to the test anxiety model predicting MA scores adds significant variance, $F(1,45)=1$. $85, p=0.18$. As a result, we chose not to use a combined model of test and trait anxiety, and instead chose to use the residuals accounting for test anxiety in measuring math anxiety. Although participants were originally recruited into high and low math anxious groups, controlling for test anxiety creates a continuous measure of math anxiety, and thus these analyses are conducted measuring math anxiety as a continuous factor. We refer to HMA and LMA individuals only as signifier for individuals who tend to be at the higher and lower ends of the MA spectrum. Additional analyses with raw MARS scores, test anxiety, trait anxiety, gender, and MA controlling for trait anxiety, were also conducted as exploratory analyses but will not be discussed in this manuscript (see Supplementary Material).

Analyses conducted with linear mixed models $\left(\mathrm{LMMs}^{2} ; \mathrm{R}\right.$ packages: Bates, Mächler, Bolker, \& Walker, 2015; Kuznetsova, Brockhoff, \& Christensen, 2017) were conducted by setting up a base model. This base model included stimulus type (math, analogy) and switching category (switch, nonswitch) as fixed factors. We evaluated the effects of these factors accounting for random effects for each participant. Then, to evaluate the full model, MA was added as an additional factor and compared with the base model to test whether MA interacted with any of the other fixed factors and accounted for additional variance in the model.

\section{Results}

We will first evaluate our main hypotheses with relation to executive function and task switching. We investigated these EF task-switching hypotheses in relation to behavior, including both response time and accuracy data, and then evaluated

\footnotetext{
${ }^{2}$ We did not correct across analyses for multiple comparisons, so $p>0.01$ should be interpreted with caution.
} 
the brain data, first evaluating these hypotheses specifically within an arithmetic network meta-ROI, and then using a whole-brain analyses to address what other regions of the brain might be active during these contrasts. Then, we evaluated our WM load hypotheses (difficulty) using a similar sequence of analyses: first evaluating the behavioral effects of increasing WM load in the math task, and then exploring how increasing WM load affects brain data in the arithmetic metaROI.

\section{Executive Function: Task Switching}

Task Switching We first evaluated how working memory changes associated with math anxiety were exacerbated by shifting and switching back and forth between sequences of math and analogy trials. In this paradigm, we expect low math anxious (LMA) individuals to show normative patterns of behavioral responses as demand on EF resources is increased by switching. For LMA individuals, increasing working memory load should be associated with increased processing time and increasing neural activity needed to address the increased demand of the problem. During task-switching, we would expect increased EF demand during initial trials in a sequence, or "switch" trials due to adopting a new set. For LMA participants who initially switch to a math trial, we would expect to observe increased response time on these trials and increased neural activity due to increased processing demands of switching to a new task (Friedman \& Miyake, 2017; Liefooghe et al., 2008; Miyake \& Friedman, 2012). For HMA individuals, we expect that this normative pattern of results should be altered, due to working memory changes associated with increased anxiety, perhaps showing a pattern of avoidant behavior. We would predict a three-way interaction between math anxiety, task-switching, and stimulus type. For HMA individuals, we would expect that switching into a math task from the analogy task would create additional demand on executive function resources due the increased anxiety experienced for math, but not necessarily for analogy trials. Thus, we would expect that in addition to the demand on executive function created by shifting to a new set, HMA individuals might experience additional working memory demand on these trials created by disruption created by increased anxiety specifically about math. Over the course of a sequence of trials, this initial anxiety might abate, and thus we predict specific changes in behavior for "math switch" trials for HMA individuals, due to increased demand on executive function created by anxiety about that specific task.

In terms of the neural pattern, we would expect to observe that brain activity during the switch and non-switch trials parallels the pattern of results found in behavior. For example, for LMA individuals, we would expect increased activity in neural regions supporting arithmetic in the switch trials to reflect increased executive function demand created by shifting. However, for HMA individuals, because anxiety leads to avoidance which disrupts math-related processing, we would expect to see decreased neural activity especially in response to an unexpected switch to math trials.

\section{Executive Function: Task Switching-Behavioral Analyses}

Task-Switching: Response time For behavioral analyses, we utilized linear mixed models (LMMs) to evaluate the impact of stimulus type (2: analogy, math), switch category (2: switch trials, nonswitch trials). For the base model, stimulus type and switch category were evaluated as fixed factors, and random intercepts were used to account for within-subject differences (random effects). We consider this to be our base model. We then evaluated how stimuli and switch category were associated with individual differences in MA by adding these fixed factors to the base model.

We evaluated differences in response time, first examining the effects of stimuli and switch category as the base model (random effects for each participant; REML criterion at convergence: 35.8$)$. We find a main effect of stimuli, $\chi^{2}(1)=$ 78.23, $p<0.0001$, such that participants responded more slowly to the analogy task $(M=1.78 \mathrm{~s}, S E=0.04)$ than the math task $(M=1.52 \mathrm{~s}, S E=0.04)$. There was no main effect of switch category, $\chi^{2}(1)=0.72, p=0.39$, and no two-way interaction between task and switch category, $\chi^{2}(1)=1.00$, $p=0.32$.

We then evaluated how these factors interacted with MA, using MA controlling for test anxiety (MARS residuals controlling for TAI; MA-TAI) as an additional fixed factor in addition to our base model. In this LMM (fixed factors: stimulus, switch category, MA-TAI; random factors: participant; REML criterion at convergence: 49.0 ), we again find a main effect of stimuli, $\chi^{2}(1)=80.75, p<0.00001$. We find no significant effect of switch category, $\chi^{2}(1)=0.85, p=0.35$. There was no main effect of MA-TAI on response time, $\chi^{2}(1)$ $=0.004, p=0.95$. There was no significant interaction between stimulus and switch category, $\chi^{2}(1)=0.90, p=0.35$. There was no significant interaction between stimulus type and MA-TAI on response time, $\chi^{2}(1)=0.70, p=0.40$. There was no significant interaction between switch category and MA-TAI, $\chi^{2}(1)=1.27, p=0.26$.

We find a three-way interaction between stimuli, switch category, and MA-TAI, $\chi^{2}(1)=5.20, p=0.02$ (Fig. 2). ${ }^{3}$ When examining this interaction, we observe that RTs are longer overall for the analogy condition than for the math

\footnotetext{
${ }^{3}$ Two outliers were removed from this analysis in the math condition because these values were greater than $3 \mathrm{SD}$ above the mean. The interaction remains significant regardless of whether these data points are included: $\chi^{2}(1)=4.44$, $p=0.04$, or omitted: $\chi^{2}(1)=5.20, p=0.02$.
} 


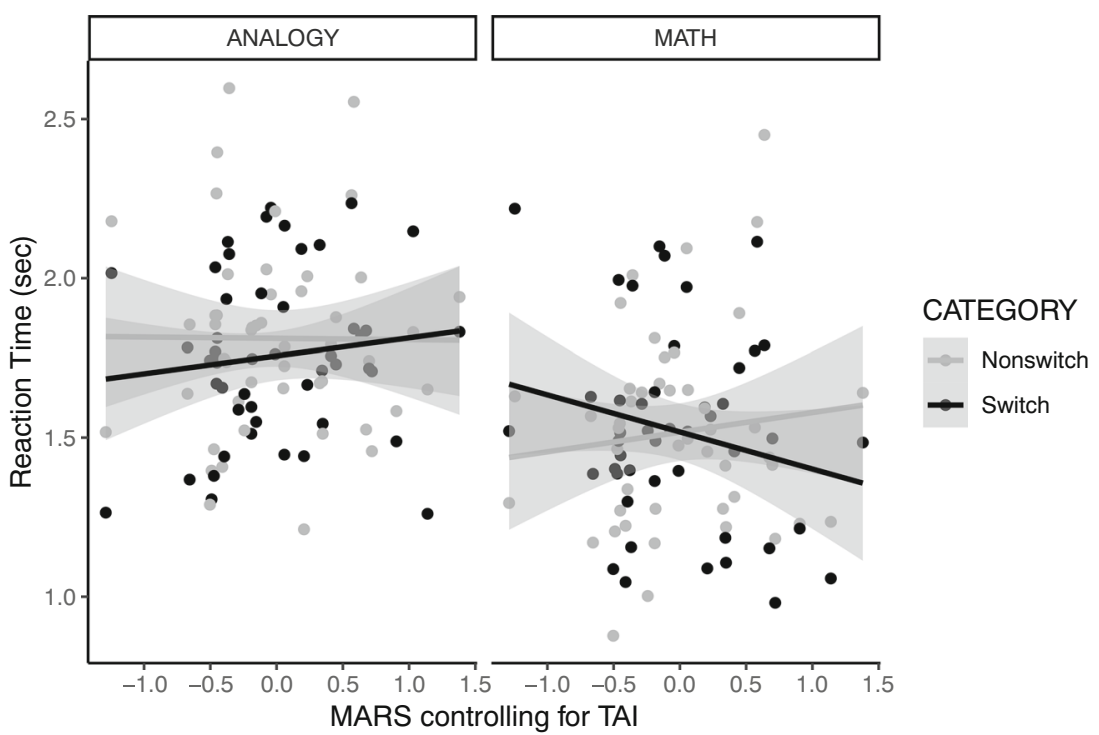

Fig. 2. Response times moderated by stimuli, switch category, and level of math anxiety (controlling for test anxiety). Three-way interaction of MA-TAI x stimuli (analogy, math) x switch category (switch, nonswitch). Individuals with increased MA speed through initial math problems (math switch trials) and spend more time on problems in the math

condition, but within the math condition, RT is determined by switch category and level of MA. LMA individuals show increased RTs during switch trials, and decreased RTs during nonswitch trials, indicating that they spend more time completing initial math problems in a sequence, but that they attenuate this switch cost over time, spending less time as the sequence continues. This normative pattern is predicted by a switch cost, such that initial trials in a sequence require more $\mathrm{EF}$ and WM resources to complete, and result in longer RTs (Friedman \& Miyake, 2017; Liefooghe et al., 2008; Miyake \& Friedman, 2012). However, HMA individuals show the opposite pattern. Individuals with increased MA show reduced RTs for math switch trials, indicating that they may speed through these initial trials. These speeded responses are consistent with previous research that suggests that HMA is associated with speed-accuracy tradeoffs (Faust et al., 1996) or initial disengagement behavior (Pizzie \& Kraemer, 2017). Interestingly, increased MA is associated with increased RTs in the math nonswitch condition, indicating that these individuals may be able to respond effectively to trials later in a sequence of math problems.

Task-switching: Accuracy We additionally evaluated differences in task accuracy using the stimuli (2: analogy, math) $\mathrm{x}$ switch category (2: switch, nonswitch) linear mixed model with random effects for each individual (REML criterion at convergence: -200.2$)$. We find a main effect of stimuli, $\chi^{2}(1)$ $=4.19, p=0.041$, such that mean accuracy is slightly higher in the analogy task $(M=72.6 \%$ correct, $S D=0.13)$ than the math task $(M=69.2 \%$ correct, $S D=0.15)$. There was no main effect of switch category, $\chi^{2}(1)=0.68, p=0.41$, such that across nonswitch trials. The opposite is true for those who have low MA, such that they spend more time on initial math trials, but attenuate this switch cost for later trials (math nonswitch trials), spending less time on these trials. As expected, both high and low MA individuals show similar RT patterns for the analogy condition.

both analogy and math conditions, switch trials $(M=0.72, S D$ $=0.15)$ did not differ from nonswitch trials $(M=0.70, S D=$ $0.13)$. There was no two-way interaction between these two stimulus types and switch category factors, $\chi^{2}(1)=2.19, p=$ 0.14 .

When we examined MA-TAI as an additional factor that might be associated with accuracy (in addition to base model; REML criterion at convergence: -177.5 ), we do not find that there was a significant effect of MA on accuracy, $\chi^{2}(1)=.98$, $p=0.32$, MA-TAI did not interact with stimuli, $\chi^{2}(1)=0.31$, $p=0.57$, did not interact with switch category, $\chi^{2}(1)=.78, p$ $=0.37$, and there was no significant three-way interaction between stimulus type, switch category, and MA-TAI on accuracy, $\chi^{2}(1)=0.35, p=0.55$. Overall, we find that math anxiety seemed to moderate response time but was not significantly associated with accuracy on switch and nonswitch trials across the math and analogy tasks. We find that overall, accuracy and response time are not significantly related to one another (please see Supplementary Material for this analysis), and for further analyses, we focused on RT.

\section{Executive Function: Task-Switching-Neuroimaging Analyses}

Task-Switching: Arithmetic meta-ROI To investigate more specifically how math anxiety correlates with mathematical processing in the brain, we selected a network of brain regions based on a meta-analysis generated by neural processing associated with arithmetic (Fig. 3A). Using a probabilistic map created by Neurosynth (Yarkoni, Poldrack, Nichols, Van Essen \& Wager, 2011; www.neurosynth.org), we selected a 

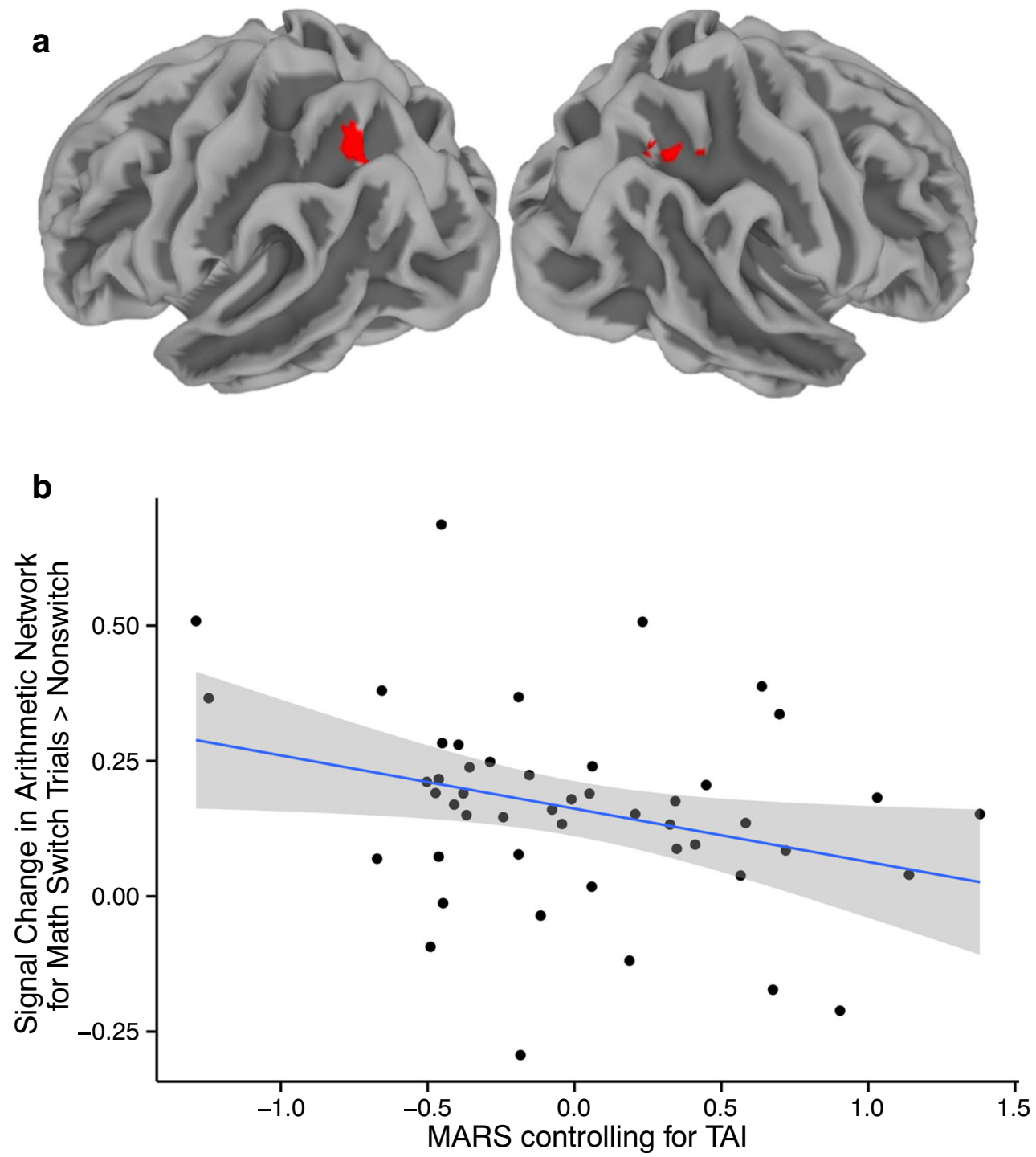

Fig. 3. Activity in the Arithmetic ROI is associated with MA. A) Network of regions from Neurosynth meta-analysis of 70+ studies generated by the query, "arithmetic" (in red). Activity pictured from the reverse-inference map is displayed at a conservative threshold of $z>6$,

network of regions of the brain associated with the search term "arithmetic" (data from 70+ studies, $\mathrm{z}>6.0$ set as a conservative threshold). Voxel clusters of this arithmetic network are listed in Table 1. This ROI is representative of bilateral activations in IPS, a region of the brain commonly associated with arithmetic processing and magnitude estimations (Dehaene, 1997; Dehaene, Spelke, Pinel, Stanescu, \& Tsivkin, 1999; Nieder \& Dehaene, 2009; Piazza, Pinel, Le Bihan, \& Dehaene, 2007), as well as other areas in frontal cortex (Fig. 3A). Using FSL's FEAT to (Jenkinson et al., 2012) to generate a t-map for each participant using the contrast of math switch $>$ math nonswitch trials, we extracted the mean activity in this arithmetic meta-ROI for each participant. Using a linear regression at this ROI, we again examined how MA is associated with neural activity indicating a high probability that activity found in these regions is associated with arithmetic processing. B) Activity in arithmetic meta-ROI is inversely correlated with math anxiety during switch trials, $F(1,46)=$ $4.85, p=0.03$, adjusted $R^{2}=0.08$.

during math switch trials compared to nonswitch trials (Fig. 3B). Importantly, we find a negative relationship between MA-TAI and neural activity during math switch trials compared to nonswitch trials, $F(1,46)=4.85, p=0.03$, adjusted $R^{2}$ $=0.08$. We observed that HMA individuals were not able to sufficiently recruit math-related regions during initial switch trials. Conversely, LMA individuals were quickly able to recruit math-related brain regions immediately upon beginning a sequence of math trials. This pattern was not observed for HMA individuals.

In order to further unpack this comparison, we compared parameter estimates in each condition compared to baseline fixation (present at the beginning and end of the runs). Although this comparison could be driven by activity in the nonswitch trials, when we compared parameter estimates 
Table 1. Clusters of activity from meta-ROI for arithmetic network

\section{Peak MNI Coordinates (mm)}

\begin{tabular}{|c|c|c|c|c|}
\hline Cluster Location & Cluster Size (voxels) & $X$ & $\boldsymbol{Y}$ & $Z$ \\
\hline Right intraparietal sulcus/superior parietal lobule & 113 & 28 & 60 & 44 \\
\hline Left intraparietal sulcus/superior parietal lobule & 38 & -32 & 52 & 46 \\
\hline Left superior parietal Lobule (posterior) & 35 & -30 & 64 & 46 \\
\hline Right Postcentral Sulcus/inferior parietal Lobule & 9 & 46 & 36 & 42 \\
\hline Right occipitoparietal cortex & 7 & 56 & 62 & 32 \\
\hline Right dorsolateral Prefrontal cortex & 6 & 22 & -22 & 2 \\
\hline Right internal capsule/white matter & 5 & 26 & -10 & 54 \\
\hline Right dIPFC & 2 & 32 & 66 & 30 \\
\hline Right occitoparietal cortex & 2 & 30 & 66 & 38 \\
\hline
\end{tabular}

All clusters reported for meta-ROI created using Neurosynth. Using a probablistic map created by Neurosynth (Yarkoni et al., 2011; www.neurosynth. org), we selected a network of regions of the brain associated with the search term "arithmetic" (data from 70+ studies, $\mathrm{z}>6.0$ set as a conservative threshold)

during math switch trials to baseline, we find a similar effect, such that low levels of MA are associated with increased activity in the arithmetic network and HMA individuals show lower levels of brain activity in this network, $F(1,46)=3.40, p$ $=0.07$, adjusted $R^{2}=0.05$. Thus, LMA individuals show increased recruitment of brain regions that support mathematical cognition during initial math trials, and these regions show differential activity for HMA individuals later in the sequence of math trials.

Furthermore, to explore this relationship, we examined how brain activity during these trials was correlated with behavior during these trials. We correlated activity during this contrast with a difference score between math switch trials and math nonswitch trials. Increased scores on this measure would be interpreted as increased RTs during the switch trials, indicating increased time taken on the switch trials compared to the nonswitch trials, which would be expected for a normative switch cost. We would hypothesize that a positive relationship would suggest increased activity in this network would be indicative of increased mathematical processing. We find a weak, positive correlation between math switch cost difference score and neural activity during the switch trials in the arithmetic network, $r(46)=0.26, p=0.07$. This relationship is not significant and should be interpreted with caution.

Task Switching: Whole Brain Analysis To further examine our hypothesis regarding neural changes associated with task switching and math anxiety, we examined the effects of math anxiety on brain processes related to math and analogy processing across trial categories. Using FSL's FEAT (Jenkinson et al., 2012), we conducted a whole-brain GLM analysis using MA-TAI as a parametric regressor to explore what other regions would be activated by task-switching in relation to MA. Clusters of activity are corrected for multiple comparisons using FSL's cluster correction algorithm and have a threshold of $z>2.3$. ( $p<0.05$, corrected). When examining math trials, we compared activity in a contrast of math switch trials to math nonswitch trials. We find an inverse relationship between MA-TAI and activity during math switch trials compared to math nonswitch trials (Fig. .4). As MA decreases, more activity is observed in the intraparietal sulcus (IPS), and a region of the lateral prefrontal cortex, as well as other regions (Table 2). Activity in this region of the IPS has been associated with mathematical processing (Dehaene, 1997; Dehaene et al., 1999; Nieder \& Dehaene, 2009). That we observe this activity associated with low MA during initial trials indicates that these participants are able to quickly recruit neural regions associated with mathematical processing when a sequence of math trials begins.

Overall, the network of regions highlighted as substrates of arithmetic processing show differential activity on the basis of MA-TAI. ${ }^{4}$ When comparing initial math trials to problems completed later in the sequence, individuals who have low levels of MA are able to quickly recruit neural regions that underlie this processing. Comparing this to the behavioral results, low MA also is associated with longer RTs for switch trials, suggesting that LMA individuals are better able to rally the neural computational resources necessary to perform these math computations when EF demand is increased due to taskswitching, exerting more effort on these problems to complete these math problems successfully. Conversely, HMA individuals' neural and behavioral responses fit a pattern of disengagement and avoidance, such that they speed through initial switch trials and show reduced neural activity during these trials.

\footnotetext{
${ }^{4}$ In investigating the specificity of this effect, we also examined a whole-brain analysis utilizing MA-TAI as a continuous predictor, this time investigating brain activity during analogy switch trials compared to non-switch trials. In this analysis, no clusters of brain activity survived cluster correction.
} 


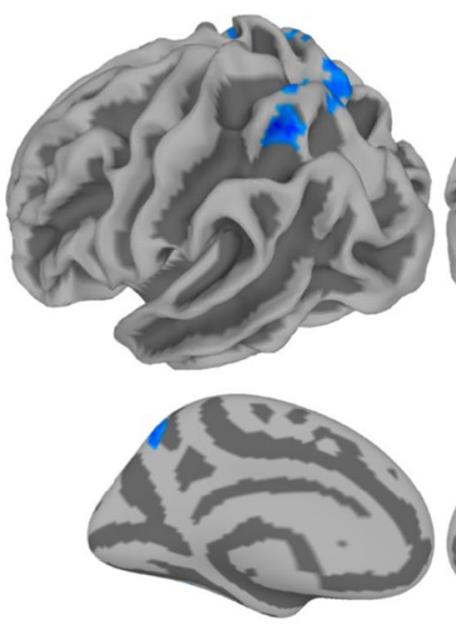

Fig.4. Math anxiety is associated with decreased activity in IPS and other regions during math switch trials. GLM results indicating brain regions in which higher levels of math anxiety were associated with lower levels of activity during initial math trials ("switch" trials) compared to the later math trials ("nonswitch" trials). Similar to what was observed in the ROI analysis (Figure 3), HMA individuals were not able to initially recruit

\section{Working Memory: Increasing Difficulty}

To unpack how math anxiety impacts WM and performance in these exploratory analyses, we investigated how math difficulty is associated with math performance as a component of EF. Just as task switching has an impact on EF resources (Liefooghe et al., 2008; Menon \& Uddin, 2010; Wylie \& Allport, 2000), we also explored how math task difficulty would be related to task performance and a neural network supporting mathematical computation (arithmetic meta-ROI). In this exploratory analysis, we only analyzed math trials, using the number of operands in the order of operations problem to determine the level of difficulty of each math problem: easy (3-operand), moderate (4-operand), and hard (5-operand). Although using the number of operands does not account for the kinds of operators used or the overall accuracy, accounting for the number of operands serves as a proxy for the amount of WM demand

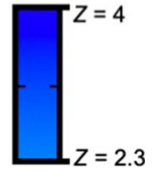

regions of the brain that might subserve successful mathematical processing (i.e., regions of the parietal lobe such as the left IPS). Clusters of activity are corrected for multiple comparisons using FSL's cluster correction algorithm and have a threshold of $\mathrm{z}>2.3 .(p<0.05$, corrected $)$ and projected on a cortical surface using AFNI's SUMA.

required to complete the problems, as each increasing operand and operator make the problems increasingly difficult.

To analyze these factors, we used LMMs for behavioral analyses with number of operands and math anxiety as fixed factors, and random effects for each individual subject and for each trial number (all models were run using individual trials instead of aggregate means as in the previous analysis, but these item-level effects were accounted for with random effects). Please see Supplementary Material for discussion of difficulty and additional discussions of amygdala ROI results.

\section{Working Memory: Increasing Difficulty-Behavioral Results}

WM Load: Response time We first evaluated a base model of response time using a LMM with fixed effect for math problem difficulty, and accounting for random effects for each

Table 2. Clusters of activity from whole brain regression using MA scores controlling for test anxiety for the contrast of Math Switch trials $>$ Math Nonswitch trials

\begin{tabular}{|c|c|c|c|c|c|c|}
\hline \multirow[b]{2}{*}{ Cluster location } & \multirow[b]{2}{*}{ Cluster size (voxels) } & \multirow[b]{2}{*}{$\boldsymbol{P}$} & \multicolumn{3}{|c|}{ Peak MNI Coordinates (mm) } & \multirow[b]{2}{*}{$\operatorname{Max} Z$} \\
\hline & & & $X$ & $\boldsymbol{Y}$ & $Z$ & \\
\hline Right Dorsal motor/Lateral parietal cortex & 1172 & $6.33 \mathrm{E}-10$ & 44 & -30 & 56 & 4.03 \\
\hline Left intraparietal sulcus & 795 & $2.38 \mathrm{E}-07$ & -28 & -58 & 48 & 4.3 \\
\hline Left Cerebellum/ Inferior occipitotemporal cortex & 612 & $6.91 \mathrm{E}-06$ & -32 & -66 & -26 & 3.91 \\
\hline Superior cerebellum & 410 & 0.000386 & 10 & -58 & -14 & 3.73 \\
\hline Right cerebellum & 351 & 0.00141 & 30 & -52 & -24 & 3.76 \\
\hline Right dorsolateral prefrontal cortex & 237 & 0.0213 & 36 & 46 & 14 & 3.74 \\
\hline
\end{tabular}

All clusters isolated by FSL cluster algorithm with a threshold of $\mathrm{Z}>2.3(p<0.05$, correct). All anatomical localizations for cluster locations were verified by searches with the peak coordinates from each cluster using Neurosynth (www.neurosynth.org) to match associations with meta-analysis maps for each location 
individual subject and individual trials (REML criterion at convergence: 4092.5). ${ }^{5}$ In this LMM, we find a main effect of difficulty (number of operands), $\chi^{2}(2)=32.95, p<0.0001$, such that RT increases as difficulty increases, for easy (3-operand, $M=1.07 \mathrm{~s}, S E=0.10$ ), moderate (4-operand, $M=1.59$, $S E=0.09$ ), and hard (5-operand, $M=1.75, S E=0.10$ ) trials. We then evaluated the base model with the addition of measures of math anxiety as additional fixed factors.

When we add MA to our base model, we find a similar effect for MA-TAI scores and difficulty evaluated as fixed factors (random effects for participants; REML criterion at convergence: 4093.5$)$. We again find a main effect of difficulty, $\chi^{2}(2)=32.42, p<0.0001$, no main effect of MA-TAI, $\chi^{2}$ (2) $=1.84, p=0.17$, and a significant interaction between difficulty and MA-TAI, $\chi^{2}(2)=9.06, p=0.01$ (Fig. 5). We find that the moderate and hard trials are roughly equivalent across the range of math anxiety but that RTs increase as MA scores increase for easy trials (similar results with alternate measures of MA are presented in the Supplementary Material).

WM Load: Accuracy We first evaluated a base model, assessing the effect of number of terms on accuracy (LMM with number of operands, i.e., difficulty, as a fixed factor, random factors accounting for participant and trial number). We find that the number of terms had a significant effect on accuracy, $\chi^{2}(2)=21.65, p<0.0001$ (REML criterion at convergence: 1967.3).

We then evaluated this model including MA-TAI as an additional fixed factor (LMM with number of operands - difficulty - and MA-TAI as fixed factors, random factors for participant and trial number; REML criterion at convergence: 1976.4). We find a main effect of difficulty on accuracy, $\chi^{2}(2)$ $=21.65, p<0.0001$, such that accuracy decreased from threeterm problems $(M=0.87, S E=0.02)$ to four-term problems $(M=0.68, S E=0.01)$ to five-term problems $(M=0.62, S E=$ 0.02 ), as predicted. The number of terms did interact with MA controlling for test anxiety, $\chi^{2}(2)=7.03, p=0.03$ (Fig. 6). As observed in Figure 6, for easy (three-term) and difficult (fiveterm) problems, we observed an expected negative relationship between MA and math task performance, such that increased MA is associated with poorer performance. However, we do not observe this relationship with the moderate (fourterm) problems, and instead observe that MA has a weak, positive relationship with task performance.

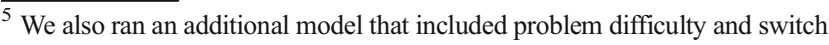
category (switch/nonswitch) as fixed factors, and random effects for each participant. Similar to the results reported here, we find a main effect of difficulty, but no main effect of switch category, $\chi^{2}(1)=0.35, p=0.55$, and no interaction between switch category and difficulty, $\chi^{2}(2)=1.56, p=0.46$. Thus, we omitted switch category as a factor in the base model and following analyses and instead focus on difficulty.
}

\section{WM Load: Neuroimaging Analyses}

For our imaging analyses, we examined how task difficulty was related to trial categories in arithmetic network. Using FSL's FEAT, we generated brain activity scaled for task difficulty using the math switch trial $>$ math nonswitch trial contrast. We extracted average activity from this map using the meta-ROI reverse-inference map representing activity associated with arithmetic (see previous). In this case, increasing percent signal change extracted for these analyses represents increased neural processing associated with increased difficulty. Therefore, a negative relationship between scores in this network and MA-TAI would indicate decreased neural activity underlying increased difficulty, which is associated with increased math anxiety. In this exploratory analysis, we examined how activity in the arithmetic network responds to additional demands on working memory associated with increasing problem difficulty and how this interacts with MA.

WM Load: Arithmetic meta-ROI Using a linear regression, we find that MA-TAI was negatively associated with neural activity reflecting increased task difficulty during math switch versus nonswitch trials within the arithmetic meta-ROI, $F(1,46)=3.65$. $p=0.062$, adjusted $R^{2}=0.05$. This relationship is not statistically significant at the $\alpha=0.05$ level. Thus, we do not find strong evidence to suggest that increased MATAI is associated with decreased activity in the arithmetic meta-ROI underlying increased problem difficulty. Therefore, further study is required to explore these associations regarding working memory load and MA, because these results are neither robust nor reliable.

\section{Discussion}

In this experiment, we examined how two different sources of demands on executive function - task-switching and WM load - were related to math performance in individuals with varying levels of math anxiety. Our results indicate that in a network of regions associated with arithmetic processing, we can observe EF-related changes in HMA associated with switching and increased demand on WM. Overall, our results suggest that HMA individuals show a pattern of disruption, suggesting that increased math anxiety is associated with a decrease in RTs for switch trials, and decreased neural activity observed during math switch trials. Increased MA is associated with speeding through initial trials that have increased demand on EF resources. Individuals with increased MA show a decrease in neural activity associated with math computations. Activity in this arithmetic network supports more advantageous mathematical computations in LMA individuals compared with those high in MA. Increasing EF demand 


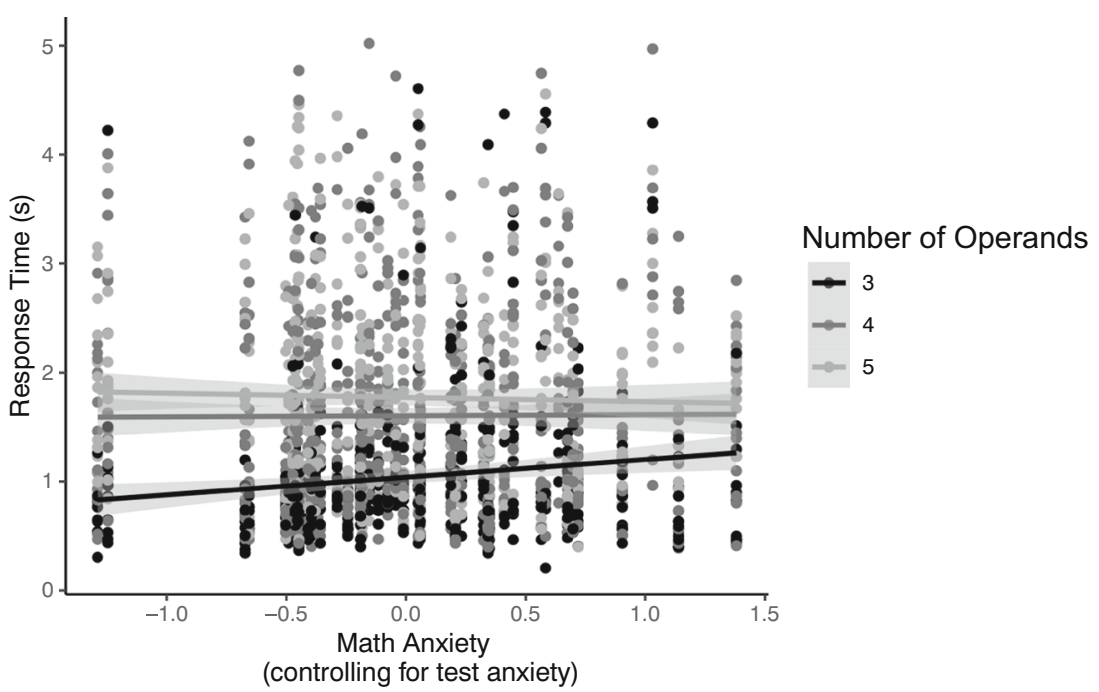

Fig. 5. Response time in math task is moderated by difficulty and math anxiety. Response times during the math trials gradually increase across levels of difficulty, but this increase occurs differently for HMA and LMA individuals, depicted here based on MA-TAI. We find that the largest differences between groups occur for the easiest problems, such that

(switching) is associated with increased neural activity in LMA individuals.

\section{Executive Functioning: Task Switching}

We find that switching is associated with disruption in response times and neural activity for HMA individuals. These individuals show abnormal disengagement behavior on initial switch trials. HMA individuals show decreased RTs during switch trials, suggesting an initial disengagement
LMA individuals are able to solve these problems particularly quickly. HMA individuals do not attenuate responses to the easier problems and spend a similar amount of time on the easy problems as they do for the more difficult moderate and hard problems.

(Pizzie \& Kraemer, 2017) by speeding through trials (Faust et al., 1996). HMA individuals disengage from initial math problems as evidenced by their speeded responses. The resulting decrease in brain activity during these trials indicates that these avoidant behaviors undercut the ability of HMA individuals to complete these math problems successfully. LMA individuals do show differences associated with switching. For example, they show a normative switch cost on initial switch trials and attenuate their responses to later nonswitch trials. LMA individuals show an advantageous

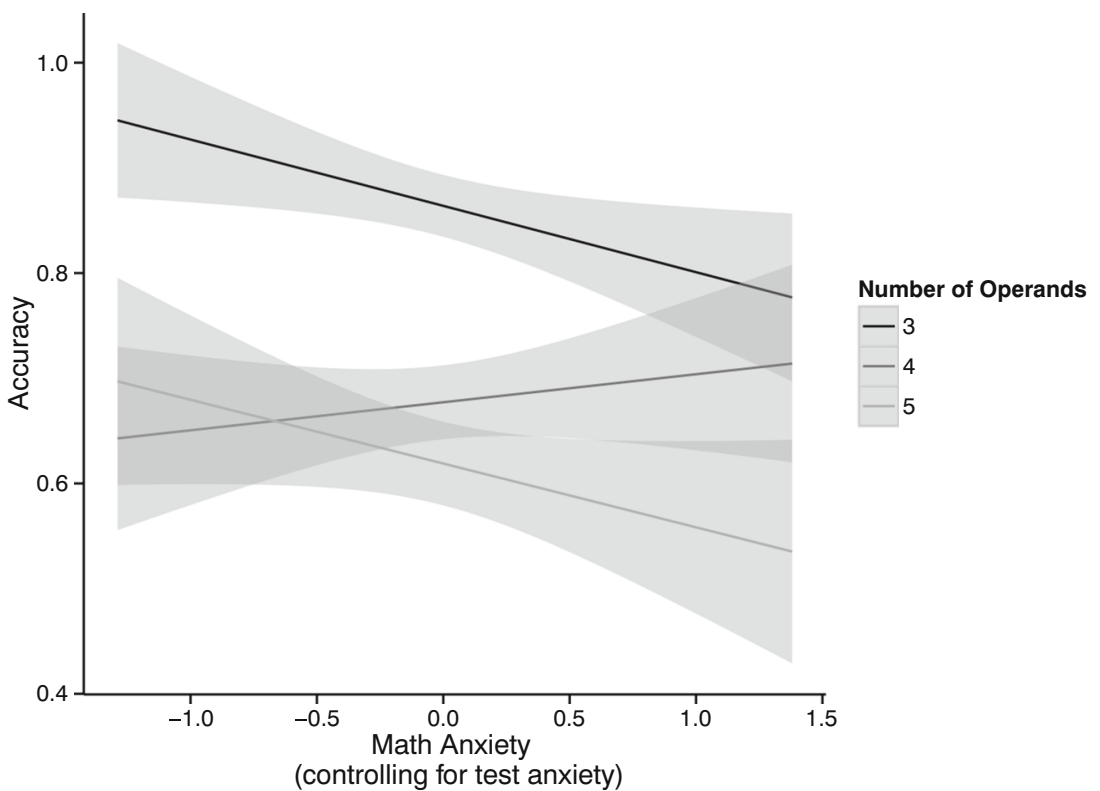

Fig.6. Relationship between MARS scores (controlling for TAI scores) and Accuracy, determined by math task difficulty (number of terms in order of operations task). Both the easy and moderate problems show a negative relationship between MA and task performance. However, for the moderate problems, we see a weak, positive relationship between MA and accuracy 
strategy during switch trials: slowing their response times during initial math trials to allow adequate processing for the increased EF demands due to switching set. Eventually, LMA individuals attenuate their responses to the new set and speed up throughout a sequence of trials. LMA individuals do not experience additional decrements associated with EF demands created by anxiety. However, HMA individuals slow for later trials, perhaps showing increased engagement on math trials presented later in a sequence (nonswitch trials).

During switch trials, LMA individuals show increased activity in regions of the brain associated with mathematical processing compared to HMA individuals. This is evidenced by increased activity in a meta-ROI specifically selected to be associated with arithmetic processing. Our results suggest that LMA individuals can more effectively recruit computational neural resources on initial trials. Our results suggest that neural resources may be diverted away from the arithmetic network for HMA individuals (see supplementary results for results related to amygdala reactivity).

Overall, we observed that HMA individuals show processing decrements in conditions associated with increased EF demand created by switching and fail to recruit neural substrates of mathematical computation that would help them effectively solve the problem. This supports the idea that increased MA is associated with disruption of cognitive resources that would otherwise be associated with math computations, but are instead distracted by intrusive thoughts, or disengaged in avoidance. On the other hand, LMA individuals are able initially to devote adequate cognitive resources to solving math problems, and show increased neural activity supporting increased mathematical processing.

\section{WM Load: Increased Difficulty}

In this exploratory analysis, we examined how additional demands placed on EF by increased WM load (i.e., updating), here indexed by increasing problem difficulty, were associated with math anxiety. Our results indicate that increased WM load created by increased difficulty exacerbates anxietyrelated differences. The hypothesis that HMA individuals show increased demands on EF and WM associated with anxiety is evident when we examine response times for increasing difficulty across math trials. HMA individuals show exaggerated responses to the easy math problems across the sequence of trials and show increased RTs. This response is perhaps indicative of increased WM load even for easy problems, whereas LMA individuals are able to quickly solve them. This pattern was somewhat counterintuitive, as we hypothesized based on previous work (Ashcraft \& Kirk, 2001; Ashcraft \& Krause, 2007b; Faust et al., 1996; Hopko et al., 1998) that the differences between MA groups would emerge as difficulty increased. Instead, this result indicates that unlike LMA individuals, who are able to quickly solve the easy problems, this increased processing time for HMA individuals is indicative of increased WM load across all levels of math problems, even easy problems. These WM-related deficits occur either through task-irrelevant distraction or by using a more WM-intensive strategy, creating demands on EF function and WM demand (Beilock, 2008).

Experimental Limitations and Considerations The results of this study should be considered in light of some important limitations. First, we specifically chose to recruit individuals who were specifically high and low in self-reported math anxiety (Suinn \& Winston, 2003) in order to focus on these more extreme patterns of behavior. Indeed, our "low math anxious" group of participants may be considered to be a non-math anxious population, as these participants have extremely low scores, and are generally characterized by having more positive feelings toward mathematics. Indeed, these participants may have utilized entirely different techniques to solve these problems, such that these LMA participants may have developed "shortcuts" in order to adapt to the demands of the problems, and may have developed strategies to solve these problems that capitalized on the answer that was presented on the response screen. This LMA group should not be considered a normative sample because we believe a normative population would likely include moderate levels of math anxiety. Although when we control for other aspects of anxiety (e.g., test anxiety) this creates a continuous range of scores, it is important to note that this study has excluded the mid-range of MA scores, and we therefore are limited in understanding the behavioral and neural response patterns in individuals with moderate levels of anxiety.

Related to our considerations about the population of math anxious individuals, in this particular math task, participants did not demonstrate overall anxiety-related accuracy deficits in mathematics. Instead the behavioral differences we observed related to math anxiety were in response times and neural responses. Indeed, it may be the case that this population of individuals is relatively "high-functioning" math anxious individuals, who are still able to perform mathematics with an improved level of accuracy, despite differences in response times and neural responses. Furthermore, we do not observe differences in accuracy across the switch and non-switch trials utilized for these analyses; however, when all trials are included in the analysis (see Supplementary Material), we do observe that high math anxious individuals have lower accuracy on the math task than low math anxious individuals, replicating previous work on math anxiety (Ashcraft, 2002; Ramirez et al., 2018). Indeed, the anxiety-related performance deficits observed on this math task may have been more subtle, or HMA individuals may have relied on some compensatory strategies (Sharp et al., 2015), and given the population of students included in this research, we should utilize caution in determining how these results may generalize to other populations of math anxious individuals. 
In addition, although our tasks were pilot-tested to be roughly equivalent in accuracy to one another $(n=12)$ such that pilot participants had roughly equivalent accuracy in the math $\left(M_{\text {math }}=75.6 \%, S D_{\text {math }}=0.16\right)$ and analogy conditions $\left(M_{\text {analogy }}=75.5 \%, S D_{\text {analogy }}=0.22\right)$, we did find a main effect of stimulus condition on accuracy in the larger sample, such that all participants overall had higher accuracy in the math condition compared to the analogy condition. Although we observe differences between the two tasks in this population, as another measure to bolster our confidence that these tasks are roughly equivalent from the pilot data, pilot participants also rated the difficulty of the analogy and math tasks, and mean difficulty ratings (on a 1-5 scale) were similar across the two tasks. These similar ratings in difficulty further illustrate that although we may have observed slightly different means in the larger sample of participants, the difficulty of task demands are perceived to be roughly similar across both tasks. In addition, although the tasks were determined to be of relatively similar difficulty, it is possible that more highly math anxious individuals may have used different techniques and strategies than the low math anxious individuals, especially in the math task. This may have resulted in differences in metacognitive strategy (Anderson, Betts, Ferris, \& Fincham, 2011), which could be related to performance and potentially, brain activity on the basis of math anxiety. Further research could explore differences in strategy use across the spectrum of math anxiety.

The present study addresses two components of executive function in math anxious individuals: task switching, and WM load - a concept that may be considered to be closely related to updating (St Clair-Thompson and Gathercole, 2006; Friedman \& Miyake, 2017). However, there is another component of working memory, inhibition, that is also likely to be impacted by anxiety (Mogg \& Bradley, 1998) and math anxiety (Hopko et al., 1998). We hope that future work will continue to address all three of these components of executive function and how they may be impacted by math anxiety. Although this study was designed to measure two components of executive function based on a prominent model of working memory (Miyake \& Friedman, 2012; Miyake et al., 2000), there are additional models of executive function that may result in different conceptualizations or interpretations of these results. For example, we might look to the expected value of control theory (Shenhav, Botvinick, \& Cohen, 2013) to generate further hypotheses about how cognitive control plays a role in math anxiety, integrating ideas about the processes of regulation, specification, and monitoring when math anxious individuals are presented with mathematical information.

Additionally, the present manuscript was not able to address other potential mediators of the effects of math anxiety on performance of mathematics, which may have been related to our results. For example, perceived math ability, spatial thinking, and math self-concept are all important considerations when studying the associations between math anxiety, math outcomes, and brain activity. We hope that future work will examine the relationship with these mediator variables on individual variation within math anxiety.

\section{Conclusions}

The purpose of this study was to better understand how math anxiety is associated with additional factors that exacerbate WM load and demands on EF. We utilized a novel taskswitching paradigm to examine how EF changes would be associated with MA. We investigated how behavioral and neural responses during initial and later problems in the sequence would be related to added cognitive difficulty. HMA individuals show a pattern of avoidance, initially disengaging from sequences of math problems, and showing a reduced ability to recruit neural networks that would effectively subserve mathematical computations. We found that individuals with low MA can quickly and effectively recruit a network of regions associated with arithmetic processing. The results of this study support the idea that EF and WM changes associated with mathematics are a significant contributing factor in MA. These results illustrate that brain networks that support mathematical computation are sensitive to changes in EF demand created by task-switching and exacerbated by problem difficulty. These results provide further evidence for a pattern of avoidant behavior in HMA individuals. This work extends our knowledge about how math anxiety is related to neural processing of arithmetic and shows that disengagement and avoidance impact neural computations in HMA individuals. In conditions where EF and WM may be taxed by taskswitching or increasing problem difficulty, this study provides important insight into how mathematical computations in the brain are moderated by math anxiety.

Acknowledgments Financial support for this research was provided by the Dartmouth College Department of Education.

Open Practices Statement This project is registered on the open science framework: https://osf.io/edav4/. Supplementary material, deidentified data and scripts for data analysis are all available for download on this platform. Preprints of this manuscript are also available through PsyArXiv: https://psyarxiv.com/knc9f/.

\section{References}

Anderson, J. R., Betts, S., Ferris, J. L., \& Fincham, J. M. (2011). Cognitive and metacognitive activity in mathematical problem solving: prefrontal and parietal patterns. Cognitive, Affective, \& Behavioral Neuroscience, 11(1), 52-67. https://doi.org/10.3758/ s13415-010-0011-0 
Ashcraft, M. H. (2002). Math anxiety: Personal, educational, and cognitive consequences. Current Directions in Psychological Science, 11(5), 181-185. https://doi.org/10.1111/1467-8721.00196

Ashcraft, M. H., \& Kirk, E. P. (2001). The relationships among working memory, math anxiety, and performance. Journal of Experimental Psychology: General, 130(2), 224-237. https://doi.org/10.1037// 0096-3445.130.2.224

Ashcraft, M. H., \& Krause, J. A. (2007). Working memory, math performance, and math anxiety. Psychonomic Bulletin \& Review, 14(2), 1 6. https://doi.org/10.3758/bf03194059

Bates, D., Mächler, M., Bolker, B., \& Walker, S. (2015). Fitting Linear Mixed-Effects Models Using lme4. Journal of Statistical Software, 67(1). https://doi.org/10.18637/jss.v067.i01

Beilock, S. L. (2008). Math performance in stressful situations. Current Directions in Psychological Science, 17(5), 339-343. https://doi. org/10.1111/j.1467-8721.2008.00602.x

Beilock, S. L., \& Maloney, E. A. (2015). Math Anxiety: A Factor in Math Achievement Not to Be Ignored. Policy Insights From the Behavioral and Brain Sciences, 2(1), 4-12. https://doi.org/10. 1177/2372732215601438

Beilock, S. L., \& Ramirez, G. (2011). On the interplay of emotion and cognitive control: Implications for enhancing academic achievement. Psychology of Learning and Motivation, 55, 137. https:// doi.org/10.1016/B978-0-12-387691-1.X0001-4

Betz, N. E. (1978). Prevalence, distribution, and correlates of math anxiety in college students. Journal of Counseling Psychology, 25(5), 441-448. https://doi.org/10.1037//0022-0167.25.5.441

Bull, R., \& Scerif, G. (2001). Executive Functioning as a Predictor of Children's Mathematics Ability: Inhibition, Switching, and Working Memory. Developmental Neuropsychology, 19(3), 273-293. https:// doi.org/10.1207/S15326942DN1903 3

Bull, R., Espy, K. A., \& Wiebe, S. A. (2008). Short-term memory, working memory, and executive functioning in preschoolers: Longitudinal predictors of mathematical achievement at age 7 years. Mathematical Cognition, 33(3), 205-228. https://doi.org/10.1080/ 87565640

Carpenter, P. A., Just, M. A., \& Reichle, E. D. (2000). Working memory and executive function: evidence from neuroimaging. Current Opinion in Neurobiology, 10(2), 195-199. https://doi.org/10.1016/ S0959-4388(00)00074-X

Daly, J. A., \& Miller, M. D. (1975). The empirical development of an instrument to measure writing apprehension. Research in the Teaching of English, 242-249.

DeCaro, M. S., Rotar, K. E., Kendra, M. S., \& Beilock, S. L. (2010). Diagnosing and alleviating the impact of performance pressure on mathematical problem solving. The Quarterly Journal of Experimental Psychology, 63(8), 1619-1630. https://doi.org/10. 1080/17470210903474286

Dehaene, S. (1997). The number sense: How the mind creates mathematics. New York, NY: Oxford University Press

Dehaene, S., Spelke, E., Pinel, P., Stanescu, R., \& Tsivkin, S. (1999). Sources of mathematical thinking: behavioral and brain-imaging evidence. Science (New York, N.Y.), 284(5416), 970-974. https:// doi.org/10.2307/25464011 ?ref=search-gateway: e3793a486fd474eb3d745fd7e7867320

Faul, F., Erdfelder, E., Lang, A. G., \& Buchner, A. (2007). G* Power 3: A flexible statistical power analysis program for the social, behavioral, and biomedical sciences. Behavior Research Methods, 175-191

Faust, M. W., Ashcraft, M. H., \& Fleck, D. E. (1996). Mathematics anxiety effects in simple and complex addition. Mathematical Cognition, 2(1), 25-62. https://doi.org/10.1080/135467996387534

Friedman, N. P., \& Miyake, A. (2017). Unity and diversity of executive functions: Individual differences as a window on cognitive structure. Cortex, 86, 186-204. https://doi.org/10.1016/j.cortex.2016.04.023

Gross, J. J., \& John, O. P. (2003). Individual differences in two emotion regulation processes: implications for affect, relationships, and well- being. Journal of Personality and Social Psychology, 85(2), 348362. https://doi.org/10.1037/0022-3514.85.2.348

Hembree, R. (1990). The nature, effects, and relief of anxiety mathematics. Journal of Research in Mathematics Educcation, 21, 33-46. https://doi.org/10.2307/749455

Hopko, D. R., Ashcraft, M. H., Gute, J., Ruggiero, K. J., \& Lewis, C. (1998). Mathematics anxiety and working memory: support for the existence of a deficient inhibition mechanism. Journal of Anxiety Disorders, 12(4), 343-355. https://doi.org/10.1016/S0887-6185(98) 00019-X

Hopko, D. R., McNeil, D. W., Gleason, P. J., \& Rabalais, A. E. (2002). The emotional Stroop paradigm: Performance as a function of stimulus properties and self-reported mathematics anxiety. Cognitive Therapy and Research, 26(2), 157-166

Jenkinson, M., Beckmann, C. F., Behrens, T. E. J., Woolrich, M. W., \& Smith, S. M. (2012). FSL. Human Brain Mapping Journal, 62(2), 782-790. https://doi.org/10.1016/j.neuroimage.2011.09.015

Kuznetsova, A., Brockhoff, P. B., \& Christensen, R. H. B. (2017). lmerTest Package: Tests in Linear Mixed Effects Models. Journal of Statistical Software, 82(13), 1-26. https://doi.org/10.18637/jss. v082.i13

Liefooghe, B., Barrouillet, P., Vandierendonck, A., \& Camos, V. (2008). Working memory costs of task switching. Journal of Experimental Psychology: Learning, Memory, and Cognition, 34(3), 478-494. https://doi.org/10.1037/0278-7393.34.3.478

Lyons, I. M., \& Beilock, S. L. (2012a). Mathematics Anxiety: Separating the Math from the Anxiety. Cerebral Cortex, 22(9), 2102-2110. https://doi.org/10.1093/cercor/bhr289

Lyons, I. M., \& Beilock, S. L. (2012b). When Math Hurts: Math Anxiety Predicts Pain Network Activation in Anticipation of Doing Math. PLoS One, 7(10), e48076. https://doi.org/10.1371/journal.pone. $0048076 . t 003$

Maloney, E. A., Schaeffer, M. W., \& Beilock, S. L. (2013). Mathematics anxiety and stereotype threat: shared mechanisms, negative consequences and promising interventions. Research in Mathematics Education, 15(2), 115-128. https://doi.org/10.1080/14794802. 2013.797744

Menon, V., \& Uddin, L. Q. (2010). Saliency, switching, attention and control: a network model of insula function. Brain Structure and Function, 214(5-6), 655-667. https://doi.org/10.1007/s00429-0100262-0

Miyake, A., \& Friedman, N. P. (2012). The Nature and Organization of Individual Differences in Executive Functions. Current Directions in Psychological Science, 21(1), 8-14. https://doi.org/10.1037/ a0014657

Miyake, A., Friedman, N. P., Emerson, M. J., Witzki, A. H., Howerter, A., \& Wager, T. D. (2000). The Unity and Diversity of Executive Functions and Their Contributions to Complex "Frontal Lobe" Tasks: A Latent Variable Analysis. Cognitive Psychology, 41(1), 49-100. https://doi.org/10.1006/cogp.1999.0734

Miyake, A., Friedman, N. P., Rettinger, D. A., Shah, P., \& Hegarty, M. (2001). How are visuospatial working memory, executive functioning, and spatial abilities related? A latent-variable analysis. Journal of Experimental Psycholgy General, 130(4), 621-640

Mogg, K., \& Bradley, B. P. (1998). A cognitive-motivational analysis of anxiety. Behaviour Research and Therapy, 36(9), 809-848. https:// doi.org/10.1016/s0005-7967(98)00063-1

Nieder, A., \& Dehaene, S. (2009). Representation of Number in the Brain. Annual Review of Neuroscience, 32(1), 185-208. https:// doi.org/10.1146/annurev.neuro.051508.135550

Núñez-Peña, M. I., Tubau, E., \& Suárez-Pellicioni, M. (2017). Post-error response inhibition in high math-anxious individuals: Evidence from a multi-digit addition task. Acta Psychologica, 177, 17-22. https://doi.org/10.1016/j.actpsy.2017.04.002

Piazza, M., Pinel, P., Le Bihan, D., \& Dehaene, S. (2007). A Magnitude Code Common to Numerosities and Number Symbols in Human 
Intraparietal Cortex. Neuron, 53(2), 293-305. https://doi.org/10. 1016/j.neuron.2006.11.022

Pizzie, R. G., \& Kraemer, D. J. (2017). Avoiding math on a rapid timescale: Emotional responsivity and anxious attention in math anxiety. Brain and Cognition, 118, 100-107. https://doi.org/10.1016/j. bandc.2017.08.004

Pizzie, R. G., \& Kraemer, D. J. (2019). The academic anxiety inventory: Evidence for dissociable patterns of anxiety related to math and other sources of academic stress. Frontiers in psychology, 9, 2684

Pizzie, R., McDermott, C., Salem, T. G., \& Kraemer, D. (2019). Neural evidence for cognitive reappraisal as a strategy to alleviate the effects of math anxiety. https://doi.org/10.31234/osf.io/84q72

Pletzer, B., Kronbichler, M., Nuerk, H. C., \& Kerschbaum, H. H. (2015). Mathematics anxiety reduces default mode network deactivation in response to numerical tasks. Frontiers in human neuroscience, 9, 202

Ramirez, G., Gunderson, E. A., Levine, S. C., \& Beilock, S. L. (2013). Math anxiety, working memory, and math achievement in early elementary school. Journal of Cognition and Development, 14(2), 187-202

Ramirez, G., Shaw, S. T., \& Maloney, E. A. (2018). Math Anxiety: Past Research, Promising Interventions, and a New Interpretation Framework. Educational Psychologist, 53(3), 145-164. https://doi. org/10.1080/00461520.2018.1447384

Sharp, P. B., Miller, G. A., \& Heller, W. (2015). Transdiagnostic dimensions of anxiety: Neural mechanisms, executive functions, and new directions. International Journal of Psychophysiology, 98(2), 365377. https://doi.org/10.1016/j.ijpsycho.2015.07.001

Shenhav, A., Botvinick, M. M., \& Cohen, J. D. (2013). The Expected Value of Control: An Integrative Theory of Anterior Cingulate Cortex Function. Neuron, 79(2), 217-240. https://doi.org/10.1016/ j.neuron.2013.07.007

Spielberger, C. D. (2009). State-Trait Anxiety Inventory. In I. B. Weiner \& W. E. Craighead (Eds.), The Corsini Encyclopedia of Psychology (pp. 1-1). Hoboken, NJ, USA: John Wiley \& Sons, Inc. https://doi. org/10.1002/9780470479216.corpsy0943
Spielberger, C. D. (1980) Test anxiety inventory. https://doi.org/10.1002/ 9780470479216.corpsy0985

St Clair-Thompson, H. L., \& Gathercole, S. E. (2006). Executive functions and achievements in school: Shifting, updating, inhibition, and working memory. The Quarterly Journal of Experimental Psychology, 59(4), 745-759. https://doi.org/10.1080/ 17470210500162854

Suárez-Pellicioni, M., Núñez-Peña, M. I., \& Colomé, À. (2015). Math anxiety: A review of its cognitive consequences, psychophysiological correlates, and brain bases. Cognitive, Affective, \& Behavioral Neuroscience https://doi.org/10.3758/s13415-015-0370-7

Suinn, R. M., \& Winston, E. H. (2003). The Mathematics Anxiety Rating Scale, a brief version: psychometric data. Psychological Reports, 92(1), 167-173. https://doi.org/10.2466/pr0.2003.92.1.167

Supekar, K., Iuculano, T., Chen, L., \& Menon, V. (2015). Remediation of Childhood Math Anxiety and Associated Neural Circuits through Cognitive Tutoring. Journal of Neuroscience, 35(36), 1257412583. https://doi.org/10.1523/JNEUROSCI.0786-15.2015

Watson, D., Clark, L. A., \& Tellegen, A. (1988). Development and validation of brief measures of positive and negative affect: the PANAS scales. Journal of Personality and Social Psychology, 54(6), 10631070. https://doi.org/10.1037/0022-3514.54.6.1063

Wylie, G., \& Allport, A. (2000). Task switching and the measurement of "switch costs." Psychological Research, 63(3-4), 212-233. https:// doi.org/10.1007/s004269900003

Yarkoni, T., Poldrack, R. A., Nichols, T. E., Van Essen, D. C., \& Wager, T. D. (2011). Large-scale automated synthesis of human functional neuroimaging data. Nature Methods, 8(8), 665-670. https://doi. org/10.1038/nmeth.1635

Young, C. B., Wu, S. S., \& Menon, V. (2012). The Neurodevelopmental Basis of Math Anxiety. Psychological Science, 23(5), 492-501. https://doi.org/10.1093/cercor/bhp063

Publisher's note Springer Nature remains neutral with regard to jurisdictional claims in published maps and institutional affiliations. 